\title{
Propagation of Blood Clotting in the Complex Biochemical Network of Hemostasis Is Described by a Simple Mechanism
}

\author{
Matthew K. Runyon, ${ }^{\dagger}$ Bethany L. Johnson-Kerner, ${ }^{\dagger}$ Christian J. Kastrup, ${ }^{\dagger}$ Thuong G. Van Ha, $^{\ddagger}$ and \\ Rustem F. Ismagilov*, \\ Department of Chemistry and Institute for Biophysical Dynamics, The University of Chicago, 929 East 57th Street, \\ Chicago, Illinois 60637, and Department of Radiology, The University of Chicago Hospitals, \\ 5841 South Maryland Avenue, MC 2026, Chicago, Illinois 60637
}

Received April 13, 2007; E-mail: r-ismagilov@uchicago.edu

This paper describes a possible mechanism responsible for regulating propagation of blood clotting from one vessel to another. The network of hemostasis controls blood clotting through a series of approximately 80 reactions. ${ }^{1}$ One of the main functions of this network is to initiate ${ }^{2}$ and localize blood clotting at sites of vascular injury. When this network fails to function correctly, extensive clot propagation beyond the site of damage can lead to thrombosis and, subsequently, to significant clinical sequelae such as heart attacks and strokes. Though investigations of the mechanisms that regulate a localized blood clotting response are the focus of substantial research effort, ${ }^{3,4}$ we are still left with the question, "what limits the size of a clot formed? That is, why do blood clots not continue to expand until all of the blood is involved?"4

One approach to understanding the regulatory mechanisms of hemostasis, as for any complex biochemical network, is to develop models of the network. ${ }^{5-7}$ Models of hemostasis have to take into account multiple parameters, including the kinetics of interrelated reactions and the influence of surfaces and flow on those reactions. ${ }^{5,8}$ Many different types of models of hemostasis have been developed, ranging from very detailed ${ }^{5}$ to very simplified. ${ }^{6}$ Detailed models that attempt to incorporate all of the parameters of the reaction network may provide valuable predictions at the level of individual biochemical components of the network. Simplified models that use only a few parameters to represent the network as a whole may provide a better representation of the overall spatiotemporal dynamics of the network. We previously described ${ }^{2,9}$ a simple chemical model, that used inorganic reactions ${ }^{10,11}$ to mimic the dynamics of hemostasis. This model was based on a simple regulatory mechanism-a threshold response ${ }^{12}$ caused by the competition between production and removal of activators (Figure 1). This threshold response is manifested by clotting occurring only when the concentration of activators, $C_{\text {act }}$, exceeds a critical concentration, $C_{\text {crit }}$. This mechanism made two predictions: (1) a clot propagates as a reactive front ${ }^{13}$ with a constant velocity, $F_{\mathrm{v}}$ [ $\left.\mathrm{m} \mathrm{s}^{-1}\right]$, if $C_{\text {act }}$, remains above $C_{\text {crit }}$, and (2) for a given geometry of vessels, clot propagation from an obstructed vessel into an unobstructed vessel with flowing blood is dependent on the shear rate, $\dot{\gamma}\left[\mathrm{s}^{-1}\right]$, in the vessel with flowing blood.

Here, we developed a microfluidic system with phospholipidcoated channels that offers precise control of flow, geometry, and surfaces. We used this system with human blood plasma to test the predictions of the proposed mechanism and demonstrated that this simple mechanism provides insight into the regulation of the spatiotemporal dynamics of clot propagation.

To test the prediction that $\dot{\gamma}$ of flowing blood regulates clot propagation, we designed a microfluidic device that exposed the

\footnotetext{
$\dagger$ The University of Chicago.

\$ The University of Chicago Hospitals.
}

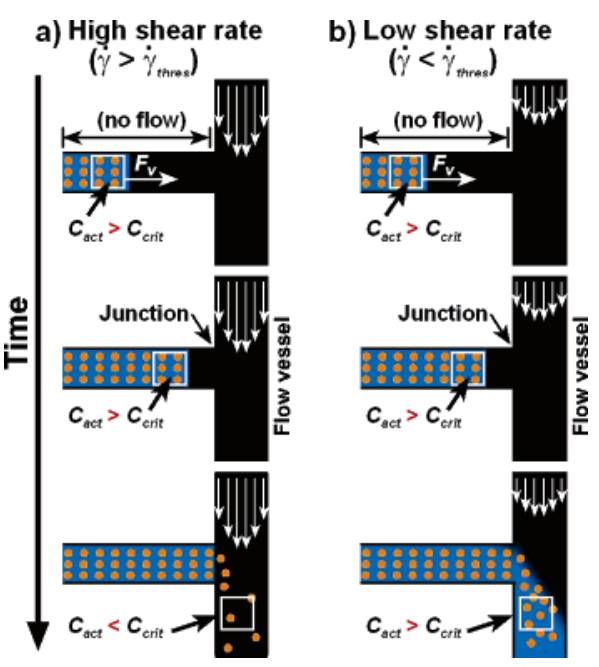

Figure 1. Schematic drawing of the proposed mechanism for regulation of clot propagation through a junction of two vessels at high (a) and low (b) shear rates. Clotting (blue) initiates when the concentration of activators (orange dots), $C_{\text {act }}$, exceeds a critical concentration, $C_{\text {crit }}$. This clot propagates through an obstructed vessel as a reactive front with a velocity, $F_{\mathrm{v}}\left[\mathrm{m} \mathrm{s}^{-1}\right]$, when $C_{\text {act }}$ remains above $C_{\text {crit }}$. When the propagating clot reaches a junction between two vessels (junction), propagation either stops or continues, depending on the shear rate, $\dot{\gamma}\left[\mathrm{s}^{-1}\right]$, in the vessel with flowing blood (flow vessel) at the junction. (a) Clot propagation stops at a junction when $\dot{\gamma}$ in the flow vessel is above the threshold shear rate, $\dot{\gamma}_{\text {thres }}$, because activator in the flow vessel is removed from the growing clot rapidly, maintaining $C_{\text {act }}$ in the flow vessel below $C_{\text {crit }}$. (b) Clot propagation continues through the junction when $\dot{\gamma}$ in the flow vessel is below $\dot{\gamma}_{\text {thres }}$, because activator in the flow vessel is removed from the growing clot slowly, causing $C_{\text {act }}$ in the flow vessel to exceed $C_{\text {crit }}$.

leading edge of a clot to flowing, recalcified blood plasma. This device allowed clot initiation in the absence of flow in one channel (initiation channels, blue in Figure 2a) without causing initiation in the unobstructed connecting channel with flowing blood plasma (flow channel, black in Figure 2a and see Supporting Information). In addition, this device incorporated a geometry in the flow channel similar to a venous valve to reproduce the recirculating flow observed in valves ${ }^{14}$ ("valve" Figure $2 a$ ). This "valve" increased the residence time of the blood plasma in the flow channel ${ }^{15}$ and allowed monitoring of clot propagation from the junction between the initiation channels and the flow channel (subsequently referred to as the junction). Control experiments confirmed recirculating flow in the "valve" (see Supporting Information).

This system also allowed control of the average flow velocity, $V_{\mathrm{av}}\left[\mathrm{m} \mathrm{s}^{-1}\right]$, and $\dot{\gamma}$. We analyzed clot propagation through a junction in terms of $\dot{\gamma}$, a parameter commonly used when studying clot formation in the presence of flow. ${ }^{5,17}$ In pressure-driven flows, the local flow rate, $V\left[\mathrm{~m} \mathrm{~s}^{-1}\right]$, at a surface is zero. Shear rate describes 


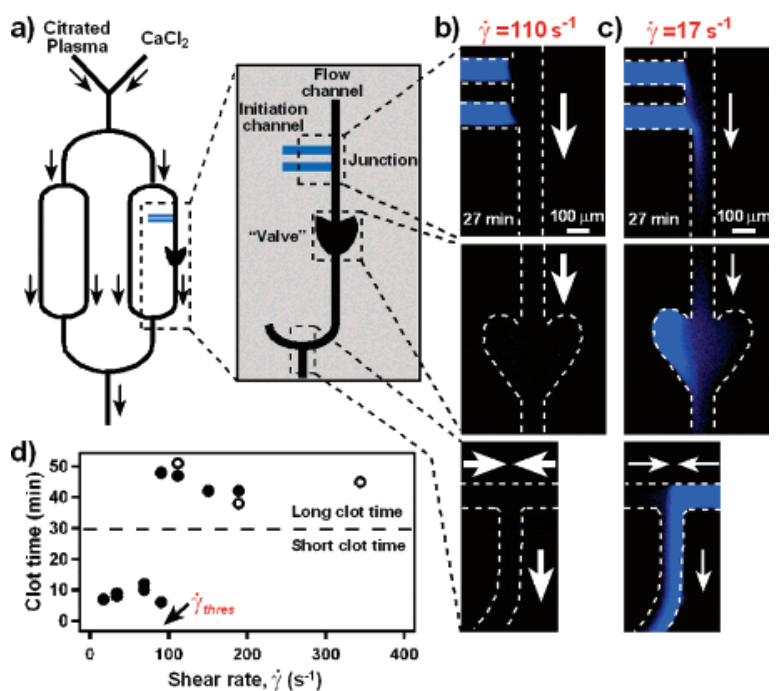

Figure 2. A threshold to $\dot{\gamma}$ regulates clot propagation through the junction. (a) Schematic drawing of the microfluidic device used to test the dependence of clot propagation through the junction on $\dot{\gamma}$. Clot propagation through the junction was determined by monitoring three regions (dashed boxes) in the flow channel (black). Clotting was monitored by thrombin-induced cleavage of a peptide-modified coumarin dye. ${ }^{16}$ Arrows indicate the direction of flow. $(b, c)$ Fluorescence microphotographs of the three regions of the flow channel $27 \mathrm{~min}$ after the clot (blue) reached the junction at high (b) and low (c) $\dot{\gamma}$. (d) Quantification of the dependence of clot propagation on $\dot{\gamma}$. Solid circles represent experiments where clotting was observed in the "valve". Open circles represent experiments stopped prior to clotting in the "valve".

the change in $V$ with increasing distance from a surface and determines transport in all directions near a surface. ${ }^{18}$ We calculated $\dot{\gamma}$ at the midpoint of the vertical wall for channels with rectangular cross sections, as described previously. ${ }^{19}$ In our experiments, a clot time was considered "long" when the time for the clot to propagate from the junction to the "valve" was greater than 30 min (dashed line, Figure 2d). Spontaneous clotting occurred in 60-80 min in the flow channel.

Propagation from the initiation channels to the "valve" of the flow channel showed a threshold response to $\dot{\gamma}$, with a threshold shear rate, $\dot{\gamma}_{\text {thres }}$, of $\sim 90 \mathrm{~s}^{-1}$ under these conditions (Figure $2 \mathrm{~d}$ ). Clotting was initiated in the absence of flow (see Supporting Information) in the initiation channel (blue in Figure 2) and propagated to the junction. As predicted, clots propagated to the junction as a reactive front with a constant velocity, $F_{\mathrm{v}} \approx 20 \mu \mathrm{m}$ $\mathrm{s}^{-1}$. When $\dot{\gamma}$ in the flow channel was above $\dot{\gamma}_{\text {thres }}$, clot propagation stopped at the junction, resulting in a long clot time (Figure 2b). However, when $\dot{\gamma}$ in the flow channel was below $\dot{\gamma}_{\text {thres, }}$ the clot in the initiation channel propagated through the junction, first to the "valve" of the flow channel and then to the rest of the flow channel downstream of the "valve", resulting in a short clot time (Figure 2c). In two experiments at $\dot{\gamma}$ close to $\dot{\gamma}_{\text {thres }}$ we observed both short and long clot times (Figure 2d), which demonstrated the sensitivity of propagation through a junction to $\dot{\gamma}$.

Localization of clots to sites of vascular damage is a key function of the complex network of hemostasis, and it has been challenging to describe by using either detailed simulations (which may be difficult to implement and interpret at the intuitive level), or simple models (which may be may be difficult to connect to specific molecules involved). We showed that combining a simple chemical mechanism $9,10,13$ can be used to make testable predictions about the spatiotemporal dynamics of the network. We experimentally confirmed these predictions using microfluidics. This simplified system does, however, impose some limitations on these experiments. For example, phospholipid-coated surfaces were used as a substitute for the endothelial cells of an intact vessel wall, constant laminar flow was used instead of pulsating flow observed in veins, and platelet poor plasma was used instead of whole blood. However, this microfluidic system combines the advantages of in vivo and in vitro techniques by allowing precise control of clot location, shear rate, and channel geometry. Confirming these results in vivo would validate the use of simplified chemical models and microfluidics to understand other complex biochemical networks., ${ }^{2,7}$

Acknowledgment. This work was funded by the ONR (Grant N000140610630), the Camille Dreyfus Teacher-Scholar Awards Program, and the NSF CAREER Award (CHE-0349034). M.K.R. was supported in part by Burroughs Wellcome Fund Interfaces I.D. 1001774. R.F.I. is a Cottrell Scholar of Research Corporation and an A. P. Sloan Research Fellow. Some of this work was performed at the MRSEC microfluidic facility (funded by the NSF). We thank Jessica M. Price for contributions in editing and writing this manuscript.

Supporting Information Available: Detailed procedure for experiments and movie comparing the recirculation in the "valve" at high and low shear rates. This material is available free of charge via the Internet at http://pubs.acs.org.

\section{References}

(1) Basmadjian, D.; Sefton, M. V.; Baldwin, S. A. Biomaterials 1997, 18, 1511-1522.

(2) Kastrup, C. J.; Runyon, M. K.; Shen, F.; Ismagilov, R. F. Proc. Natl. Acad. Sci. U.S.A. 2006, 103, 15747-15752.

(3) (a) Giesen, P. L. A.; Rauch, U.; Bohrmann, B.; Kling, D.; Roque, M.; Fallon, J. T.; Badimon, J. J.; Himber, J.; Riederer, M. A.; Nemerson, Y. Proc. Natl. Acad. Sci. U.S.A. 1999, 96, 2311-2315. (b) Falati, S.; Gross, P.; Merrill-Skoloff, G.; Furie, B. C.; Furie, B. Nat. Med. 2002, 8, 11751180. (c) Panteleev, M. A.; Ovanesov, M. V.; Kireev, D. A.; Shibeko, A M.; Sinauridze, E. I.; Ananyeva, N. M.; Butylin, A. A.; Saenko, E. L.; Ataullakhanov, F. I. Biophys. J. 2006, 90, 1489-1500. (d) Dorffler-Melly, J.; de Kruif, M.; Schwarte, L. A.; Franco, R. F.; Florquin, S.; Spek, C. A.; Ince, C.; Reitsma, P. H.; ten Cate, H. Basic Res. Cardiol. 2003, 98 347-352. (e) Smith, S. A.; Morrissey, J. H. J. Thromb. Haemostasis 2004, 2, 1155-1162. (f) Mann, K. G.; Brummel, K.; Butenas, S. J. Thromb. Haemostasis 2003, 1, 1504-1514.

(4) Morrissey, J. H. J. Thromb. Haemostasis 2003, 1, 878-880.

(5) Kuharsky, A. L.; Fogelson, A. L. Biophys. J. 2001, 80, 1050-1074.

(6) Beltrami, E.; Jesty, J. Proc. Natl. Acad. Sci. U.S.A. 1995, 92, 87448748

(7) (a) Winfree, A. T. Science 1994, 266, 1003-1006. (b) Janes, K. A.; Albeck, J. G.; Gaudet, S.; Sorger, P. K.; Lauffenburger, D. A.; Yaffe, M. B. Science 2005, 310, 1646-1653.

(8) Diamond, S. L. Biophys. J. 2001, 80, 1031-1032.

(9) Runyon, M. K.; Johnson-Kerner, B. L.; Ismagilov, R. F. Angew. Chem. Int. Ed. 2004, 43, 1531-1536.

(10) Nagypal, I.; Epstein, I. R. J. Phys. Chem. 1986, 90, 6285-6292.

(11) Horvath, A. K.; Nagypal, I.; Peintler, G.; Epstein, I. R. J. Am. Chem Soc. 2004, 126, 6246-6247.

(12) Mihaliuk, E.; Sakurai, T.; Chirila, F.; Showalter, K. Phys. Rev. E 2002 65.

(13) Hanna, A.; Saul, A.; Showalter, K. J. Am. Chem. Soc. 1982, 104, 38383844.

(14) Karino, T.; Motomiya, M. Thromb. Res. 1984, 36, 245-257.

(15) Shelby, J. P.; Lim, D. S. W.; Kuo, J. S.; Chiu, D. T. Nature 2003, 425, $38-38$.

(16) Kawabata, S. I.; Miura, T.; Morita, T.; Kato, H.; Fujikawa, K.; Iwanaga, S.; Takada, K.; Kimura, T.; Sakakibara, S. Eur. J. Biochem. 1988, 172, $17-25$.

(17) Tseng, P. Y.; Jordan, S. W.; Sun, X. L.; Chaikof, E. L. Biomaterials 2006, 27, 2768-2775.

(18) Ismagilov, R. F.; Stroock, A. D.; Kenis, P. J. A.; Whitesides, G.; Stone, H. A. Appl. Phys. Lett. 2000, 76, 2376-2378.

(19) Nataraja, N.; Lakshman. S Indian J. Tech. 1973, 10, 435-438. JA072602P 